\section{Impacto de los problemas bucales sobre la calidad de vida de niños}

Abanto JA, Bönecker M, Raggio DP. Impacto de los problemas bucales sobre la calidad de vida de niños. Rev Estomatol Herediana. 2010; 20(1):38-43.

\section{RESUMEN}

El concepto de calidad de vida relacionada a la salud bucal (CVRSB) se refiere al impacto que la salud o enfermedad bucal tiene en las actividades diarias de los individuos, en su bienestar o calidad de vida. Diversos instrumentos de medición de CVRSB fueron desarrollados en los últimos años para evaluar el impacto de los problemas bucales en la salud física, mental y social de los individuos. Los niños también fueron considerados en estos estudios, visto que éstos son afectados por numerosos desórdenes bucales y orofaciales tales como, caries dental, traumatismos dentales, maloclusiones y anomalías craneofaciales. Por tal motivo, el objetivo de este artículo fue describir los instrumentos desarrollados, las percepciones de niños y padres sobre CVRSB y realizar una revisión sobre el estado clínico bucal de los niños y su calidad de vida.

Palabras clave: CALIDAD DE VIDA / SALUD BUCAL / NIÑO.

Impact of oral health problems on children's quality of life ABSTRACT

The concept of oral health-related quality of life (OHRQoL) relates to the impact which ora health or disease has on the individual's daily functioning, well-being or life quality. Numerous OHRQoL measures have been developed in recent years to assess the impact of oral health problems on individuals' physical, mental and social health. Children were also considered in those studies, as they are affected by numerous oral and orofacial disorders, such as dental caries, traumatic dental injuries, malocclusions and craniofacial anomalies. Thus, the purpose of this paper was to describe the instruments developed, the perceptions in children and parents on QHRQoL and review children clinical oral status and their quality of life.

Key words: QUALITY OF LIFE / ORAL HEALTH / CHILD.

\section{Jenny Abanto Alvarez ${ }^{1}$ \\ Marcelo Bönecker ${ }^{2}$ \\ Daniela Prócida Raggio ${ }^{3}$}

${ }^{1}$ Especialista, Máster y Doctoranda en

Odontopediatría.

2Profesor Titular del Departamento de Ortodoncia y Odontopediatría.

3Profesora Asociada del Departamento de Ortodoncia

y Odontopediatría.

Facultad de Odontología de la Universidad de São Paulo - FOUSP.

\section{Correspondencia}

\section{Daniela Prócida Raggio}

Faculdade de Odontologia de São Paulo,

Departamento de Odontopediatria

Avenida Professor Lineu Prestes, 2227 - Cidade

Universitária.

CEP: 05508-000. São Paulo - SP - Brasil.

Teléfono: (11) 3091-7835 Fax: (11) 3091-7854

e-mail: danielar@usp.br

Recibido :25 de setiembre de 2010

Aceptado : 10 de enero de 2010

\section{Introducción}

La salud bucal forma parte de la salud general y es esencial para la Calidad de Vida (CV). Todos los individuos deben disponer de una condición de salud bucal que les permita hablar, masticar, reconocer el sabor de los alimentos, sonreír, vivir libres de dolor e incomodidad y relacionarse con otras personas sin apremio.

Los indicadores clínicos bucales utilizados en la clínica odontológica han sido tradicionalmente restrictos a personas con síntomas tales como dolor, incomodidad y alteraciones estética (1). Todavía no es una práctica común, para el diagnóstico y plan de tratamiento, analizar el impacto que estos indicadores tienen en la vida de las personas, principalmente de niños. Es por ese motivo que a lo largo de las dos últimas décadas fueron realizados diversos estudios en niños relacionados al indicador conocido como CVRSB - Calidad de Vida Relacionada a la Salud Bucal, que en inglés es descrito como COHRQoL - Child Oral Health Related Quality of Life (2).

La calidad de vida puede ser definida como "la percepción del individuo sobre su posición en la vida, en el contexto de la cultura $y$ del sistema de valores en los cuales este vive, y en relación a sus objetivos, expectativas, padrones y preocupaciones" (3). El concepto de calidad de vida, además de comprender una representación subjetiva de la sensación de bienestar, es multidimensional e incluye tanto dimensiones positivas como negativas.

La literatura relata la existencia de algunos instrumentos disponibles capaces de medir el impacto funcional y psicosocial de las enfermedades bucales sobre la $\mathrm{CV}$ de las personas. Sin embargo, la mayoría fue desarrollada para adultos y ancianos, habiendo así una carencia de instrumentos específicos para niños. Éstos últimos constituyen el mayor foco de la Salud Pública Odontológica, reflejando la necesidad urgente de la aplicación del concepto de CVRSB en esta fase de la vida.

\section{Percepción de niños y padres sobre la CVRSB}

Las teorías y estudios en psicología indican que existe un proceso continuo del desarrollo cognitivo, emocional, social y lingüístico durante la infancia, siendo que la estructura del autoconcepto y la concepción de salud de los niños dependen de la edad (4).

De acuerdo con la psicología del desarrollo infantil, la edad de seis años marca el inicio del pensamiento abstracto y del autoconcepto $(4,5)$. A esta edad, comienzan a comparar sus características físicas y trazos de personalidad con las de otros niños. Su capacidad de realizar juicios sobre su apariencia, calidad de las amistades, pensamientos de otras personas, emociones $\mathrm{y}$ comportamiento se desenvuelve 
gradualmente a través de la mitad de la infancia (6-10 años de edad) $(4,5)$. A esta edad la idea de estética asociada a la salud comienza a ser incorporada en la mente del niño y pasa a ser integrada a su concepto de autoestima (5).

Gradualmente, los niños desarrollan la capacidad de utilizar un amplio espectro de estímulos internos para identificar sus enfermedades. Aproximadamente a los 11 ó 12 años de edad, éstos visualizan la salud como un concepto multidimensional organizado alrededor de las siguientes construcciones: funcionalidad, adhesión a comportamientos de aparente buen estilo de vida, sentido general de bienestar y relaciones con los otros (5). La forma como estos conceptos son organizados varían de acuerdo con la edad y con el tipo de experiencias a las cuales el niño estuvo expuesto en su vida (5). De acuerdo con la literatura, la comprensión de estos conceptos también es aparentemente afectado por el género del niños $(6,7)$, siendo las niñas las que más relatan una $\mathrm{CV}$ afectada (8).

Actualmente, existen en la literatura algunos instrumentos para evaluar la CVRSB de niños escolares (con más de 6 años de edad). Estos instrumentos fueron destinados para ser aplicados en niños con diversas condiciones bucales y dentofaciales, y para ser ajustados a las diferencias de desarrollo entre niños de diversas edades. Sin embargo, la medición de la CVRSB en niños preescolares (menores de 6 años de edad) aún envuelve algunos problemas metodológicos, tales como, alteraciones en la capacidad de comprensión del niño a esta edad y dificultad para separar las percepciones del niño de las de sus padres $(9,10)$. A pesar de que los instrumentos desarrollados para medir la CV deberían ser respondidos por los propios niños, que tienen el derecho de expresar sus opiniones y de hacerlas respetar, en el caso de preescolares los instrumentos son generalmente respondidos por los responsables. Los relatos ofrecidos por los padres sirven de gran valor a la hora de evaluar la CVRSB en preescolares. Se entiende que, mientras más graves sean las alteraciones patológicas, más estrecha será la relación entre padres e hijos, de forma que esos estén más familiarizados con las actividades y sentimientos de sus hijos (9).

Las condiciones bucales no tienen sólo impacto directo en los niños, sino también un efecto indirecto en los padres y miembros de la familia. Las evidencias indican que, por ejemplo, los cuadros de caries resultan en pérdidas de días laborables de los padres que tienen que quedarse en casa para cuidar a sus hijos o gastar tiempo y dinero en el acceso a los cuidados dentarios (11). Así, estas influencias sobre la familia también son importantes de ser investigadas como parte de la evaluación de la CVRSB.

Basados en los resultados de una revisión sistemática (9), se puede afirmar que por medio de cuestionarios con técnicas adecuadas, es posible obtener informaciones válidas y confiables, no sólo de los niños como también de los padres, aunque éstos no compartan necesariamente visiones similares sobre CVRSB. A pesar de que los relatos de los padres puedan estar incompletos debido a la falta de conocimiento sobre determinadas experiencias de sus hijos, estos continúan ofreciendo y complementando informaciones muy útiles. Así, datos valiosos pueden ser perdidos si no fueran colectados.

Hoy en día, la forma más adecuada de integrar los relatos de los padres con los de los niños permanece siendo un gran desafío para los investigadores del área de CVRSB.

\section{Instrumentos utilizados para medir CVRSB en niños}

Los instrumentos utilizados para medir CVRSB generalmente son construidos bajo la forma de cuestionarios compuestos por preguntas que intentan medir, por medio de respuestas organizadas bajo la forma de escalas numéricas, cuanto los aspectos de la vida de las personas, en los dominios de síntomas orales, limitaciones funcionales, bienestar emocional y social, actividades diarias, condiciones psicológicas, entre otros, son afectados por las condiciones de salud $(12,13)$. Así, un aspecto importante de ese instrumento es su capacidad de representar bajo forma numérica las diferencias entre personas y comunidades con relación a la CVRSB (12).

Actualmente, los cuestionarios existentes para medir la CVRSB en niños y cuya validad y confiabilidad fueron comprobados en estudios pueden ser divididos en cuestionarios respondidos por los padres o responsables y cuestionarios respondidos por los propios niños (Tabla 1). De los instrumentos citados en la Tabla 1 sólo el ECOHIS - Early Childhood Oral Health Impact Scale (20) y el Child Oral Impacts on Daily Performances Index (Child-OIDP) (21) fueron adaptados transculturalmente $\mathrm{y}$ validos en el idioma español, siendo que la mayoría de esos ya fueron validados en diversos idiomas. 
Tabla 1. Instrumentos validados para medir Calidad de Vida Relacionada a la Salud Bucal en niños.

\begin{tabular}{|c|c|c|c|}
\hline Referencia & Nombre del instrumento & $\begin{array}{c}\text { Entrevis- } \\
\text { tado }\end{array}$ & $\begin{array}{c}\text { Edad albo } \\
\text { (años) }\end{array}$ \\
\hline $\begin{array}{l}\text { Locker et al., } \\
2002 \text { (14) }\end{array}$ & $\begin{array}{l}\text { Family Impact Scale (FIS) } \\
\text { Parental/Caregivers }\end{array}$ & Padres & $\begin{array}{l}\text { Padres de niños } \\
\text { entre } 2 \text { a } 14 \text { años } \\
\text { de edad } \\
\text { Padres de niños }\end{array}$ \\
\hline $\begin{array}{l}\text { Jokovic et al., } \\
2003 \text { (15) }\end{array}$ & $\begin{array}{l}\text { Perceptions Questionnaire } \\
\text { (P-CPQ) }\end{array}$ & Padres & $\begin{array}{l}\text { entre } 6 \text { a } 14 \text { años } \\
\text { de edad }\end{array}$ \\
\hline $\begin{array}{l}\text { Pahel et al., } \\
2007 \text { (16) }\end{array}$ & $\begin{array}{l}\text { Early Childhood Oral } \\
\text { health Scale (ECOHIS) }\end{array}$ & Padres & $\begin{array}{l}\text { entre } 2 \text { a } 5 \text { años } \\
\text { de edad }\end{array}$ \\
\hline Jokovic et al., & Child Perceptions & & Niños entre 6 y \\
\hline $2002(2)$ & Questionnaire (CPQ6-7) & Niños & 7 años de edad \\
\hline Jokovic et al., & Child Perceptions & & Niños entre 8 y \\
\hline $2004(17)$ & Questionnaire (CPQ8-10) & Niños & 10 años de edad \\
\hline Jokovic et al., & Short Child Perceptions & & Niños entre $11 \mathrm{y}$ \\
\hline 2006 (18) & $\begin{array}{l}\text { Questionnaire (CPQ11-14) } \\
\text { Child Oral Impacts on }\end{array}$ & Niños & 14 años de edad \\
\hline $\begin{array}{l}\text { Gherunpong et } \\
\text { al., } 2004(19)\end{array}$ & $\begin{array}{l}\text { Daily Performances Index } \\
\text { (Child-OIDP) }\end{array}$ & Niños & $\begin{array}{l}\text { Niños entre } 11 \text { y } \\
14 \text { años de edad }\end{array}$ \\
\hline
\end{tabular}

Impacto de las principales enfermedades bucales $y$ condiciones socioeconómicas en la CVRSB de niños

Impacto de la caries dental

La caries dental es considerada la enfermedad más común de la infancia. La Organización Mundial de la Salud (22) ha estimado que $60 \%$ a $90 \%$ de todos los niños escolares están afectados por esta enfermedad habiendo un aumento directamente proporcional con el aumento de la edad.

Fue hipotetizado que niños con mayor experiencia de caries dental podrían tener un mayor impacto sobre su CVRSB y la de sus padres (16). El impacto negativo de la caries dental sobre la vida de los niños incluye: mayor experiencia de sensación de dolor, dificultad para masticar, disminución del apetito, pérdida de peso, dificultad para dormir, alteración en el comportamiento (irritabilidad y baja autoestima), pérdida de días de escuela y/o disminución del rendimiento escolar (23-26).
Los estudios que miden la CVRSB en niños con caries dental muestran que esta está correlacionada con todos los dominios evaluados en los cuestionarios, siendo que las puntuaciones más altas se encontraron en los dominios de síntomas orales, seguido por el de limitaciones funcionales y por último los de bienestar emocional y social (27-29).

\section{Impacto de la maloclusión dentaria}

Los estudios sugieren que el impacto más significativo de la maloclusión en la CV es psicosocial $(8,30)$, en vez de condiciones producidas por las condiciones bucales tales como síntomas orales y limitaciones funcionales. Además, la dificultad de sonreír debido a la mala posición de los dientes ha sido considerada unos de los motivos de mayor impacto en la CVRSB de niños (31).

De acuerdo con O'Brien (30) y Abanto (26), algunos cuestionarios no fueron específicamente desarrollados para medir el impacto de los problemas oclusales y, algunas de las preguntas en los dominios de síntomas y limitaciones bucales no son necesariamente relevantes para pacientes escolares y preescolares con maloclusión.

\section{Impacto de los Traumatismos} Dentarios

Hasta el presente momento solamente un estudio evaluó el impacto de los traumatismos dentarios sobre la CV de niños escolares (32). En este estudio, no fue encontrado impacto negativo en la CVRSB en la presencia de traumatismos leves y sí un impacto negativo en los niños que presentaron traumas más graves. Con relación a preescolares, la alteración de color en los dientes antero-superiores ha mostrado tener un impacto negativo en la vida de los niños, principalmente en lo que se refiere al dominio de auto-imagen y autoestima (26).

La Tabla 2 muestra los principales resultados encontrados en estudios que evaluaron el impacto de diversas condiciones bucales en la CV de niños.

\section{Impacto de las condiciones} socioeconómicas

Todavía existen pocos estudios mostrando que los factores socioeconómicos influyen en las respuestas sobre el impacto de diversas enfermedades en la CV $(26,32,36)$.

Un estudio que evaluó la influencia de estos factores mostró que los niños escolares provenientes de familias de bajo salario poseían una peor salud bucal y calidad de vida (32). Este autor concluyó que existen disparidades socioeconómicas durante la evaluación de 
Tabla 2. Resultados de estudios realizados sobre Calidad de Vida Relacionada a la Salud Bucal en niños.

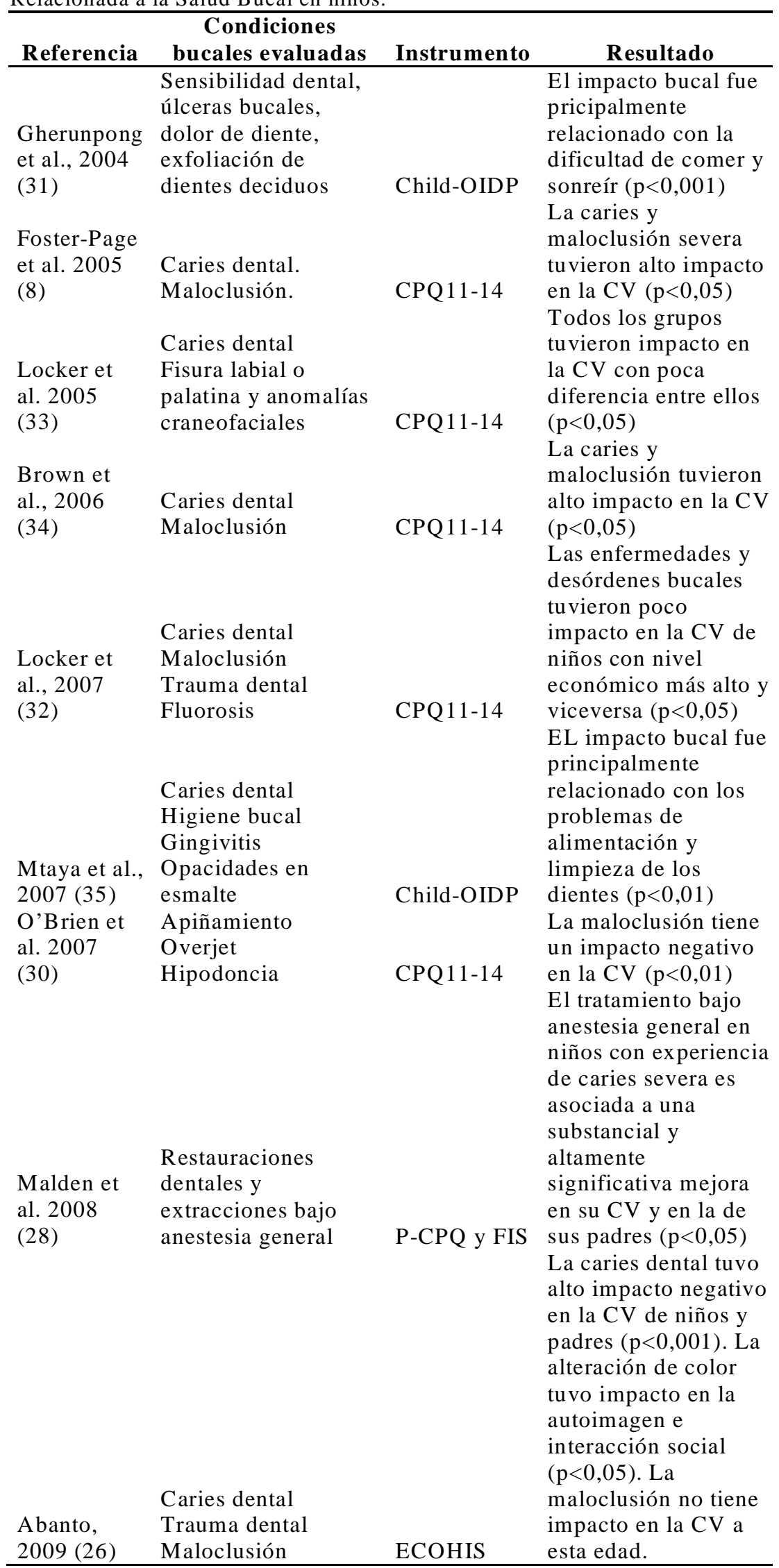

la CVRSB y que el salario familiar puede ser considerado como predictor de las puntuaciones de los cuestionarios de CVRSB. En concordancia con este estudio, Abanto (26) describió que el alto salario familiar se comporta como un factor de protección para una mejor CV independiente de la presencia de enfermedades bucales tales como la caries, traumatismos dentales y maloclusiones.

A partir de estos pocos estudios se observa que existe una evidencia limitada y poco clara sobre la asociación y magnitud del efecto de las condiciones socioeconómicas en la CVRSB, principalmente en niños.

\section{Consideraciones finales}

A pesar de los progresos en el desarrollo de instrumentos para medir el impacto de las condiciones bucales en la calidad de vida de niños, aún hay mucho para ser estudiado. Todavía necesitamos conocer, por ejemplo, la sensibilidad de estos instrumentos en estudios longitudinales, qué condiciones tienen impacto significativo sobre la vida de niños preescolares, cómo el desarrollo físico/psíquico y las experiencias relacionadas a otras enfermedades influyen en la percepción del niño sobre su propia salud bucal y de que forma los tratamientos odontológicos a los cuales el niño fue sometido actúan sobre la percepción de su bienestar. Finalmente, cabe resaltar que, en este momento que la odontología basada en evidencias viene creciendo en importancia, es fundamental que, tanto en la clínica como en la investigación, sea substancialmente ampliada la utilización de los instrumentos de evaluación de la CVRSB con el fin de que los indicadores realmente importantes para la vida de los niños 
y sus familias comiencen a ser considerados.

\section{Referencias bibliográficas}

1. Marques LS, Ramos-Jorge ML, Paiva SM, Pordeus IA. Malocclusion: esthetic impact and quality of life among Brazilian schoolchildren. Am J Orthod Dentofacial Orthop. 2006; 129(3):424-7

2. Jokovic A, Locker D, Stephens M, Kenny D, Tompson B, Guyatt G. Validity and reliability of a questionnaire for measuring child oral-health-related quality of life. J Dent Res. 2002; 81(7):459-63.

3. World Health Organization. WHOQOL - measuring quality of life. The World Health Organization quality of life instruments. Geneva: World Health Organization; 1997.

4. Hetherington EM, Parke RD, Locke VO. Child Psychology: A Contemporary Viewpoint. New York: The McGraw-Hill Companies; 1999.

5. Rebok G, Riley A, Forrest C, Starfield B, Green B, Robertson J, Tambor E. Elementary schoolaged children's reports of their health: a cognitive interviewing study. Qual Life Res. 2001; 10(1):59-70.

6. Barbosa TS, Gavião MB. Oral health-related quality of life in children: part I. How well do children know themselves? A systematic review. Int J Dent Hyg. 2008; 6(2):93-9.

7. Jokovic A, Locker D, Guyatt G. How well do parents know their children? Implications for proxy reporting of child health-related quality of life. Qual Life Res. 2004; 13(7):1297-307.

8. Foster Page LA, Thomson WM, Jokovic A, Locker D. Validation of the Child Perceptions
Questionnaire (CPQ 11-14). J Dent Res. 2005; 84(7):649-52.

9. Barbosa TS, Gavião MB. Oral health-related quality of life in children: part III. Is there agreement between parents in rating their children's oral healthrelated quality of life? A systematic review. Int J Dent Hyg. 2008; 6(2):108-13.

10. Rosenbaum P, Cadman D, Kirpalani H. Pediatrics: assessing quality of life. In: Spilker B, ed. Quality of Life Assessments in Clinical Trials. New York: Raven Press, 1990:205-15.

11. Gift HC, Reisine ST, Larach DC. The social impact of dental problems and visits. Am J Public Health. 1992; 82(12):1663-8.

12. Sevenhuysen GP, TrumbleWaddell J. A new perspective on quality of life. J Clin Epidemiol. 1997; 50(3):231-2.

13. Wallander JL, Schmitt M, Koot HM. Quality of life measurement in children and adolescents: issues, instruments, and applications. J Clin Psychol. 2001; 57(4):571-85.

14.Locker D, Jokovic A, Stephens M, Kenny D, Tompson B, Guyatt G. Family impact of child oral and oro-facial conditions. Community Dent Oral Epidemiol. 2002; 30(6):438-48.

15.Jokovic A, Locker D, Stephens M, Kenny D, Tompson B, Guyatt G. Measuring parental perceptions of child oral healthrelated quality of life. J Public Health Dent. 2003; 63(2):67-72.

16. Pahel BT, Rozier RG, Slade GD. Parental perceptions of children's oral health: the Early Childhood Oral Health Impact Scale (ECOHIS). Health Qual Life Outcomes. 2007; 30(5):6.

17.Jokovic A, Locker D, Tompson
B, Guyatt G. Questionnaire for measuring oral health-related quality of life in eight- to ten-yearold children. Pediatr Dent. 2004; 26(6):512-8.

18. Jokovic A, Locker D, Guyatt G. Short forms of the Child Perceptions Questionnaire for 11-14-year-old children (CPQ1114): development and initial evaluation. Health Qual Life Outcomes. 2006; 4:4.

19.Gherunpong S, Tsakos G, Sheiham A. Developing and evaluating an oral health-related quality of life index for children; the CHILD-OIDP. Community Dent Health. 2004; 21(2):161-9.

20. Talekar BS, Rozier RG, Zeldin LP. Spanish version of the Early Childhood Oral Health Impact Scale [abstract]. J Dent Res 2005; 84(Special Issue A). Abstract no.2653.

21. Bernabé E, Sheiham A, Tsakos G. Acomprehensive evaluation of the validity of Child-OIDP: further evidence from Peru. Community Dent Oral Epidemiol. 2008; 36(4):317-25.

22. World Health Organization. WHO Oral Health Data Bank. Geneva: World Health Organization; 2002.

23. Ayhan H, Suskan E, Yildirim S. The effect of nursing or rampant caries on height, body weight and head circumference. J Clin Pediatr Dent. 1996; 20(3):20912.

24. Feitosa S, Colares V, Pinkham J. The psychosocial effects of severe caries in 4-year-old children in Recife, Pernambuco, Brazil. Cad Saude Publica. 2005; 21(5):1550-6.

25. Oliveira LB, Sheiham A, Bönecker M. Exploring the association of dental caries with social factors and nutritional 
status in Brazilian preschool children. Eur J Oral Sci. 2008; 116(1):37-43.

26. Abanto JA. Impacto das doenças e desordens bucais na qualidade de vida relacionada à saúde bucal de crianças pré-escolares e de seus pais [Dissertação]. São Paulo: Universidade de São Paulo, 2009. 95 págs.

27.Do LG, Spencer A. Oral healthrelated quality of life of children by dental caries and fluorosis experience. J Public Health Dent. 2007; 67(3):132-9.

28. Malden PE, Thomson WM, Jokovic A, Locker D. Changes in parent-assessed oral healthrelated quality of life among young children following dental treatment under general anaesthetic. Community Dent Oral Epidemiol. 2008; 36(2):10817.

29.Barbosa TS, Gavião MB. Oral health-related quality of life in children: part II. Effects of clinical oral health status. A systematic review. Int J Dent Hyg. 2008; 6(2):100-7.

30.O'Brien C, Benson PE, Marshman Z. Evaluation of a quality of life measure for children with malocclusion. $\mathrm{J}$ Orthod. 2007;34(3):185-93.

31.Gherunpong S, Tsakos G, Sheiham A. The prevalence and severity of oral impacts on daily performances in Thai primary school children. Health Qual Life Outcomes. 2004; 2:57.

32.Locker D. Disparities in oral health-related quality of life in a population of Canadian children. Community Dent Oral Epidemiol. 2007; 35(5):348-56.

33.Locker D, Jokovic A, Tompson

B. Health-related quality of life of children aged 11 to 14 years with orofacial conditions. Cleft
Palate Craniofac J. 2005; 42(3):260-6.

34. Brown A, Al-Khayal Z. Validity and reliability of the Arabic translation of the child oralhealth-related quality of life questionnaire (CPQ11-14) in Saudi Arabia. Int J Paediatr Dent. 2006; 16(6):405-11.

35. Mtaya M, Astrøm AN, Tsakos G. Applicability of an abbreviated version of the Child-OIDP inventory among primary schoolchildren in Tanzania. Health Qual Life Outcomes. 2007; 5:40.

36.Pappa E, Kontodimopoulos N, Papadopoulos AA, Niakas D. Assessing the socio-economic and demographic impact on health-related quality of life: evidence from Greece. Int $\mathrm{J}$ Public Health. 2009; 54(4):2419. 